\title{
Comparative genomics of Riemerella anatipestifer reveals genetic diversity
}

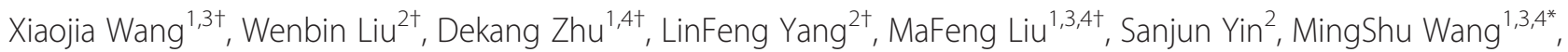
RenYong Jia ${ }^{1,3,4}$, Shun Chen ${ }^{1,3,4}$, KunFeng Sun ${ }^{1,3,4}$, Anchun Cheng ${ }^{1,3,4^{*}}$ and Xiaoyue Chen ${ }^{1,4}$

\begin{abstract}
Background: Riemerella anatipestifer is one of the most important pathogens of ducks. However, the molecular mechanisms of $R$. anatipestifer infection are poorly understood. In particular, the lack of genomic information from a variety of $R$. anatipestifer strains has proved severely limiting.

Results: In this study, we present the complete genomes of two $R$. anatipestifer strains, RA-CH-1 (2,309,519 bp, Genbank accession CP003787) and RA-CH-2 (2,166,321 bp, Genbank accession CP004020). Both strains are from isolates taken from two different sick ducks in the SiChuang province of China. A comparative genomics approach was used to identify similarities and key differences between RA-CH-1 and RA-CH-2 and the previously sequenced strain RA-GD, a clinical isolate from GuangDong, China, and ATCC11845.

Conclusion: The genomes of RA-CH-2 and RA-GD were extremely similar, while RA-CH-1 was significantly different than ATCC11845. RA-CH-1 is 140,000 bp larger than the three other strains and has 16 unique gene families. Evolutionary analysis shows that RA-CH-1 and RA-CH-2 are closed and in a branch with ATCC11845, while RA-GD is located in another branch. Additionally, the detection of several iron/heme-transport related proteins and motility mechanisms will be useful in elucidating factors important in pathogenicity. This information will allow a better understanding of the phenotype of different $R$. anatipestifer strains and molecular mechanisms of infection.
\end{abstract}

Keywords: Riemerella anatipestifer, Comparative genomics, Structural variation

\section{Background}

Riemerella anatipestifer (RA) is a Gram-negative bacterium in the family Flavobacteriaceae and rRNA superfamily $\mathrm{V}[1]$. $R$. anatipestifer can infect ducks, geese, turkeys, chickens, and other birds, and leads to a contagious septicemia [2]. Transmission between ducks occurs vertically (through the egg) as well as horizontally via the respiratory tract [3]. $R$. anatipestifer has a worldwide distribution and is one of the leading problems of the farmed duck industry, mainly infecting young ducks with a mortality of up to $90 \%$. Animals that survive infection may be stunted [4], leading to decreased production. Riemerellosis causes substantial economic losses

\footnotetext{
*Correspondence: mshwang@163.com; chenganchun@vip.163.com

${ }^{\dagger}$ Equal contributors

${ }^{1}$ Avian Disease Research Center, College of Veterinary Medicine of Sichuan Agricultural University, 46\# Xinkang Road, Ya'an, Sichuan 625014, P.R. China ${ }^{3}$ Institute of Preventive Veterinary Medicine, Sichuan Agricultural University, Chengdu, Sichuan 611130, P.R. China

Full list of author information is available at the end of the article
}

in countries with significant duck industries, such as China and Southeastern Asia [5]. While serotyping is the traditional method to differentiate $R$. anatipestifer isolates [5], other methods, including PCR based on 16S rRNA or $r p o B$ genes [6,7], repetitive-sequence polymerase chain reaction (Rep-PCR) [8], multiplex PCR [9], matrix-assisted laser desorption/ionization-time of flight (MALDI-TOF) mass spectrometry [10], plasmid profiling, pulsed-field gel electrophoresis (PFGE), and PCR-restriction fragment length polymorphism (PCR-RFLP) [11] have also been used to characterize isolates.

At least 21 serotypes have been described in different countries $[5,7,12]$ with no cross-protection between different serotypes. Among pathogenic isolates, serotypes $1,2,3,5$, and 15 are the most common [13]. Individual animals can be infected with multiple serotypes and changes in the predominant serotype from year to year within a single farm have been described [12].

Although Reimerellosis causes serious economic losses, the pathogenesis of $R$. anatipestifer and the virulence factors 
remain mostly unknown. Subramaniam et al. identified OmpA as a predominant immunogenic outer membrane protein [14]. Later, it was shown that ompA mutant strains were attenuated when used to infect ducklings, with decreased adhesion and invasion capacities in Vero cells, indicating that OmpA is a virulence factor [15]. Recently, Zhai et al. selected six proteins that cross-reacted with serotypes 1 and 2 for a vaccine trial. Only administration of the recombinant outer membrane protein A (OmpA) showed a protective effect when challenged by serotype $1(60 \%)$ and serotype $2(50 \%)$ [13]. Additionally, VapD was identified as a virulence factor, with homology to virulence-associated proteins of other bacteria [16]. CAMP cohemolysin was identified as another potential virulence factor, which may lyse red blood cells and release iron for use by the organism [17].

The publication of the first $R$. anatipestifer genome, ATCC11845 [18] has improved the understanding of the disease mechanisms underlying infection. However, the relatively limited number of published strains has hindered more in-depth analysis. In order to establish genetic differences between pathogenic strains, we sequenced two $R$. anatipestifer genomes, $\mathrm{RA}-\mathrm{CH}-1$ and $\mathrm{RA}-\mathrm{CH}-2$, which were isolated from sick ducks from Chengdu and Mianyang, respectively, in the SiChuan province of China, and compared these to the two previously sequenced strains, ATCC11845 and RA-GD (which was isolated from a sick duck in GuangDong, China).

\section{Results and discussion}

\section{General features of the $R$. anatipestifer genomes}

Genomic read-data for the two $R$. anatipestifer strains sequenced in this study were generated using a multiplexing approach in a single Illumina HiSeq lane. The resulting sequences were assembled using SOAPdenovo. The previously sequenced ATCC11845 has single 2,164,087 bp circular chromosome with $35.01 \%$ GC content. In contrast, RA-CH-1 is larger at 2,309,519 bp with $35.07 \%$ GC content while $\mathrm{RA}-\mathrm{CH}-2$ is similar at 2,166,321 bp with $35.04 \%$ GC content. ATCC11845, RA-CH-1 and RA-CH2 contain 2,091 (92.8\% of the genome), 2,236 (97.8\%), and 2,095 (97.8\%) genes, respectively. All genomes have approximately the same codon usage frequency.

\section{Genes associated with iron/hemin metabolism}

Bacteria that reside in animal tissues must acquire iron from their host for growth. A large number of genes coding for iron and hemin metabolism and iron-dependent transcriptional regulators were annotated in all sequenced strains. A total of one siderophore-interacting protein (Sip) and three siderophore receptors were detected that could be involved in $\mathrm{Fe}^{3+}$ uptake. The siderophore-interacting protein was previously found to be involved in iron utilization and mutation of this gene significantly decreased virulence in $R$. anatipestifer $\mathrm{CH}-3$ [19]. There were two putative proteins, $\mathrm{FeoA}$ and $\mathrm{FeoB}$, for $\mathrm{Fe}^{2+}$ uptake and two outer membrane hemin receptors. All sequenced strains had one extracellular hemin-binding protein (hemophore), and no hemin degrading proteins were detected via sequence analysis, suggesting that $R$. anatipestifer may have a novel hemin degrading system. Additionally, we found that several TonB-dependent receptors with a plug domain, one set of the ExbB-ExbD-TonB complex, one set of the ExbBExbD-ExbD-TonB complex, and one TonB family protein. The TonB-dependent receptor TbdR1 (Riean_1607) has been found to be involved in heme acquisition in $R$. anatipestifer. The median lethal dose of a $t b d R 1$ mutant was approximately 45 -fold higher than the wild-type $\mathrm{CH}-3$ strain [20]. Our group has confirmed the functions of TonB and the TonB complex and determined that the ExbB-ExbDTonB complex is involved in heme uptake in ATCC11845 (unpublished data).

$R$. anatipestifer is usually grown on blood-enriched media. Sequence analysis shows that $R$. anatipestifer does not encode for genes involved in heme synthesis, hemF, $Y$, and $G$ (http://www.kegg.jp/pathway/rae00860). This suggests that the heme compounds from the culture plate could be essential for growth. We have determined that $R$. anatipestifer can synthesize hemin using protoporphyrin as a substrate and subsequently use hemin as an iron source (unpublished data). However, the function of proteins involved in iron/hemin metabolism in maintaining and enhancing virulence still requires experimental investigation.

\section{Genes associated with gliding motility}

Cells of the phylum Bacteroidetes can rapidly move over surfaces using a process called gliding motility. In $F$. johnsoniae, at least nineteen genes ( $g l d A$, gldB, gldD, gldF, gldG, gld H, gldI, gldJ, gldK, gldL, gld M, gldN, sprA, sprB, sprC, sprD, sprE, sprT and $\operatorname{Rem} A$ ) involved in gliding motility have been identified [21-23]. These motility proteins constitute a novel protein secretion system, the Por secretion system (PorSS) [24], which may be an integral part of the gliding motility machinery [23]. In $F$. johnsoniae, the Por secretion system consists of gldK, gldL, gldM, gldN, sprA, sprE, and sprT, which are needed for secretion of an extracellular chitinase [23]. Similarly, the $P$. gingivalis PorSS is needed for secretion of gingipain protease virulence factors [25].

Genome analysis finds that $R$. anatipestifer encodes for several genes involved in gliding motility, including gldA, gldB, gldC, gldD, gldF, gld H, gldJ, gldK, gldL, gldM, gldN, porP, and porT. Many of the proteins encoded by these genes are predicted to localize to the cellular envelope. In F. johnsoniae, gldK, gldL, gldM, and gldN are clustered together on in two adjacent operons, although gld $K$ is transcribed separately from the other three genes [26]. A similar arrangement is found in $R$. anatipestifer 
as well as other Bacteroidetes, such as F. psychrophilum and $C$. hutchinsonii [21]. This organization suggests that the protein products of these genes work together as a part of a complex, and the extensive conservation of the genes encoding this protein secretion system indicates it is likely functional in $R$. anatipestifer. Analysis of this system in $R$. anatipestifer has the potential to provide insight into disease pathogenesis. For example, in $P$. gingivalis, the PorSS is involved in gliding motility and pathogenesis [24]. The PorSS, its relationship to gliding, and its function in pathogenesis, needs to be further studied in $R$. anatipestifer.

\section{Complete genome analysis and structural variation}

The genomes of RA-CH-2 and RA-GD were similar to the genome of ATCC11845, while the genome of RA$\mathrm{CH}-1$ is significantly different. All four strains had some deletions unique to a specific strain. The missing parts of the RA-CH-2 and RA-GD genomes were focused in three different places as shown in the colinearity analysis (Figure 1A). However, deleted sequences of RA-CH-1 were dispersed throughout the genome (Figure 1A). Moreover, there were more genome rearrangements between RA-CH-1 and ATCC11845 than the other two genomes. By analyzing the genomic coverage rate and sequence similarity of homologous regions for the four different genomes, we found that there is a higher degree of similarity between ATCC11845 and both RA$\mathrm{CH}-2$ and RA-GD than RA-CH-1 (Figures $1 \mathrm{~B}$ and $\mathrm{C}$ ). Compared to the genome of ATCC11845, RA-CH-1 had a higher SNP and indel density than RA-CH-2 and RAGD. The distribution of SNPs and indels for RA-CH-2 and RA-GD are similar (Figure 2). Furthermore, we found that the genomes of $\mathrm{RA}-\mathrm{CH}-1$ and $\mathrm{RA}-\mathrm{CH}-2$ had commons deletions compared to ATCC11845 and RAGD (Figure 3). Both RA-CH-1 and RA-CH-2 contain same inserted and deleted sequences, which suggests these deletions are localized to the region both these strains were isolated from.

\section{Functional analysis of variant genes}

Based on analysis of mutation types, we found that indels mainly induced non-synonymous mutations, while SNP primarily caused synonymous mutations. There were significantly more SNPs than indels (Figure 4). In addition, we analyzed three different types of genes using COG [27], KEGG [28], PATHWAY [29], and GO functional characterization databases [30] (Figure 5). First were genes that were found by pairing with sequences from other strains, but were not annotated because of a mutation in the original sequence. Second were structural variations (SV) region genes. Third were genes containing SNPs or

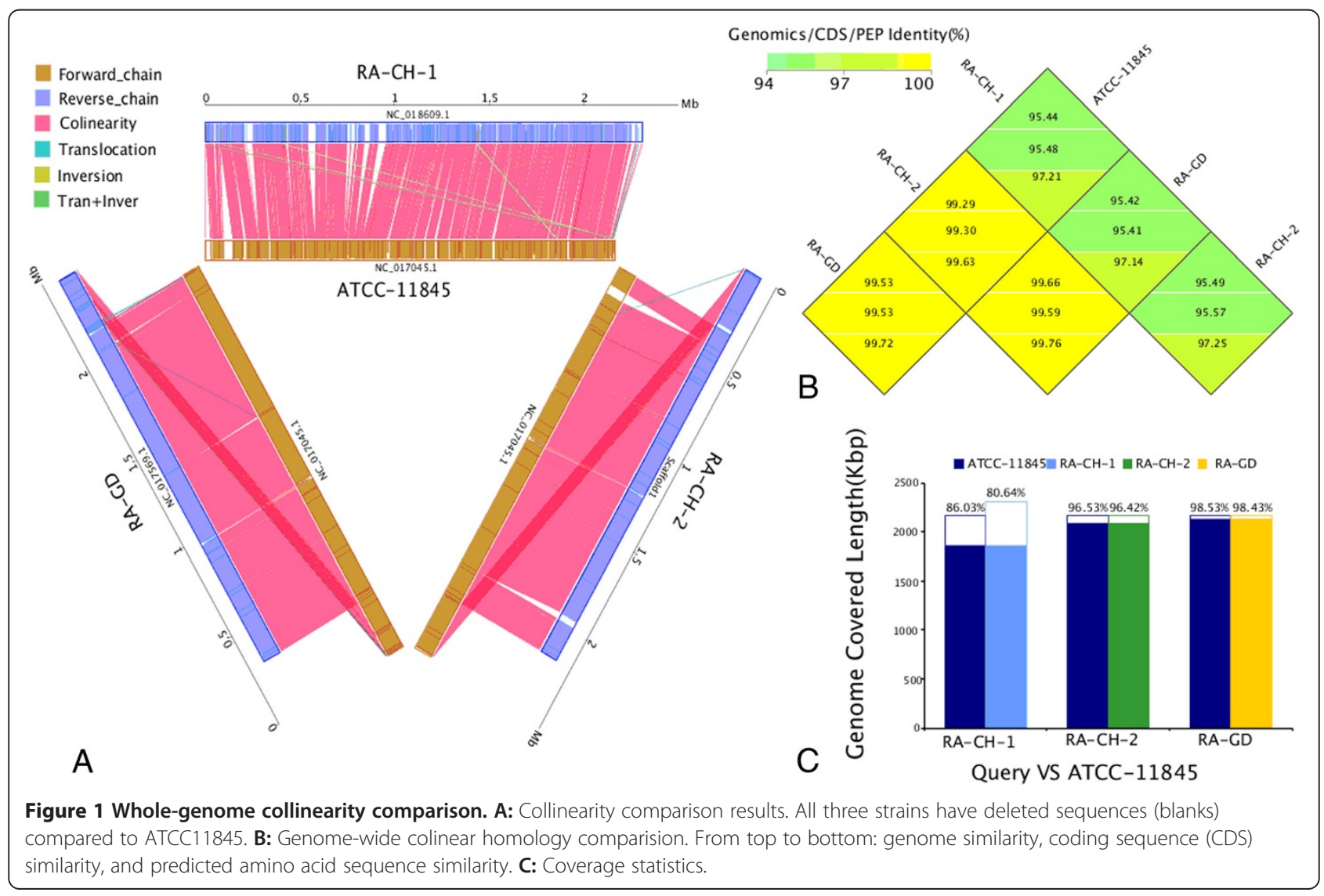




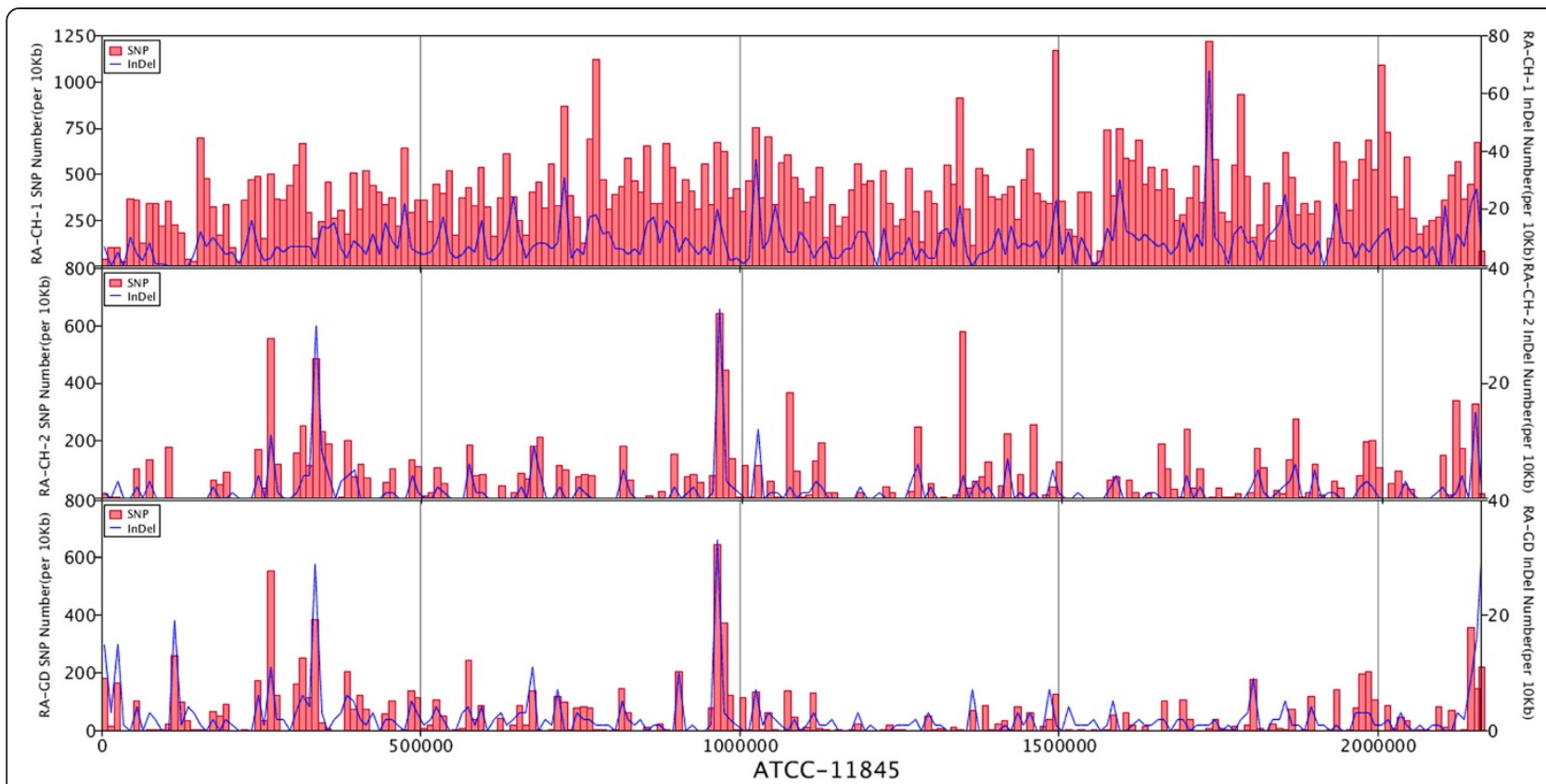

Figure 2 Genome SNP-indel maps. The distribution of SNP and indels in RA-CH-1, RA-CH-2 and RA-GD. SNP distribution is presented as a bar graph and indel distribution as a line graph.

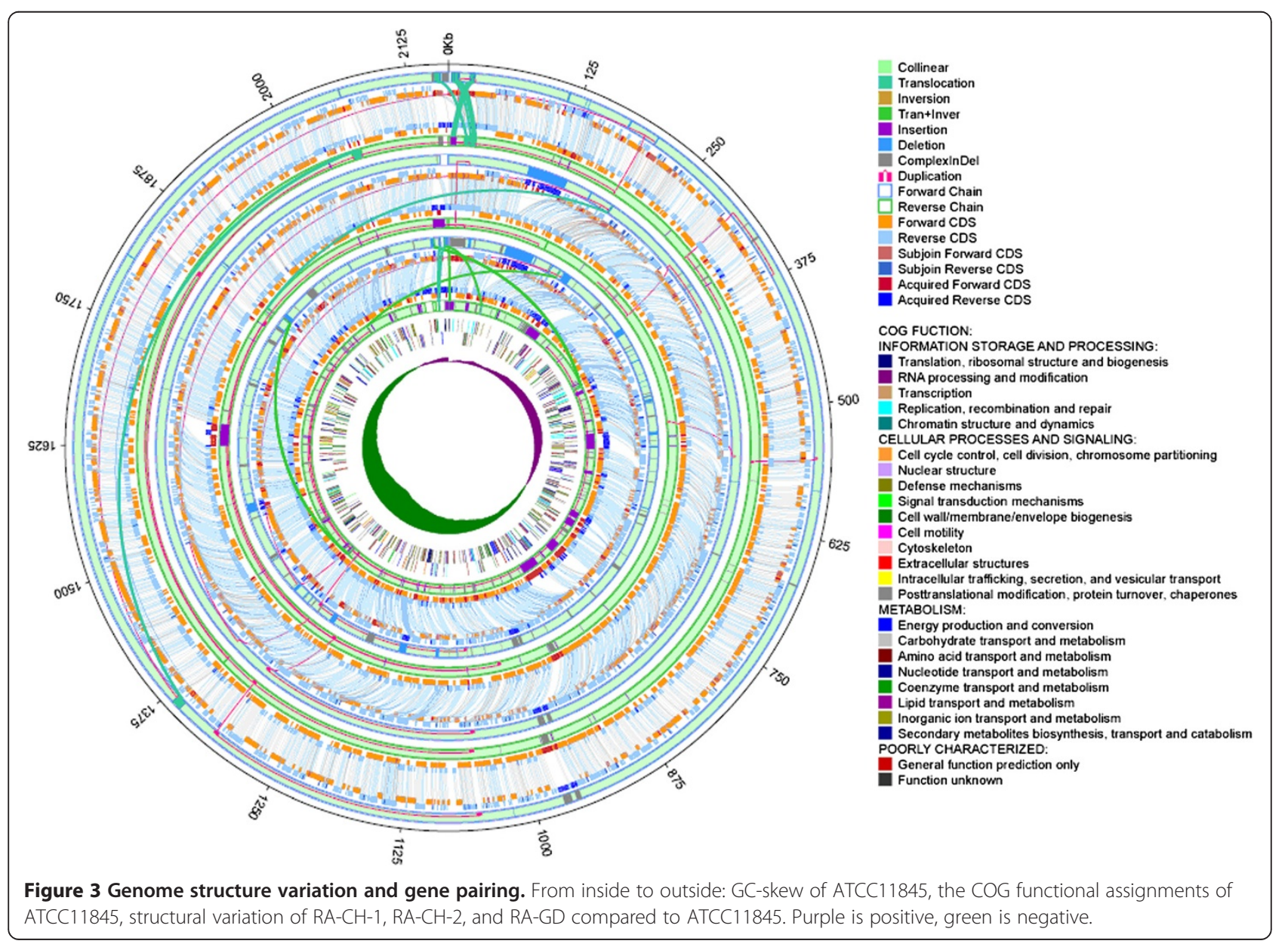




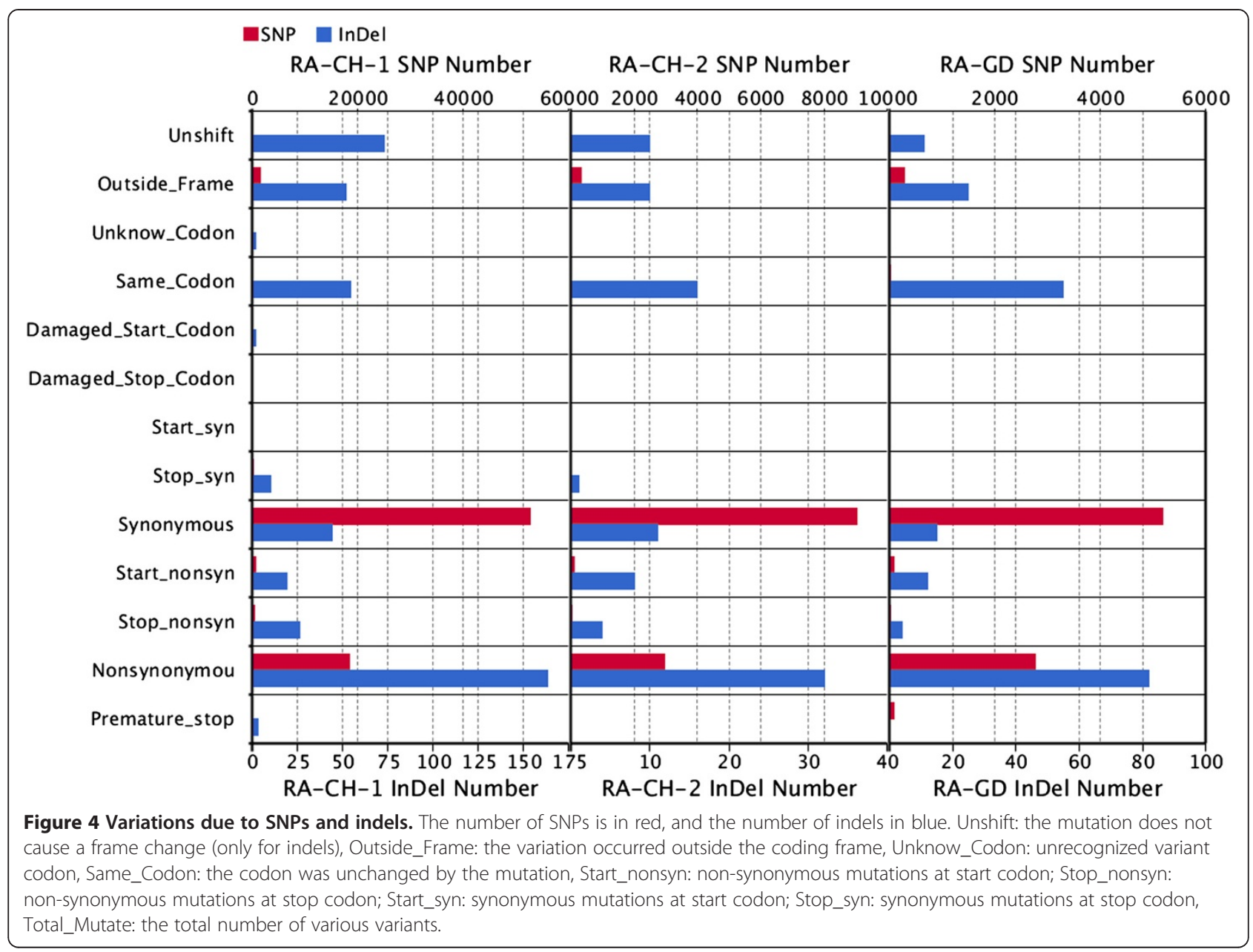

small indels. Among these three groups, the first have no significant difference in the three databases, indicating that there is no increased rates of change in any particular functional gene category or pathway.

Through COG analysis, we can find that the SV region sequences of RA-CH-1, RA-CH-2, RA-GD have no significant differences in polymorphism frequencies. RA$\mathrm{CH}-1$ had 14 areas with significantly higher rates of SNPs and indels, RA-CH-2 showed no significant differences, and RA-GD had significantly higher SNP and indel rates in the COG "M: Cell wall/membrane/envelope biogenesis", which may be associated with host invasion or antibiotic resistance. SNPs are the main type of polymorphism, indicating that $R$. anatipestifer evolution mainly relies on this rather than deletion or insertion to generate genetic diversity.

\section{Gene family cluster analysis}

A gene family is a set of several similar genes formed by duplication of a single original gene, generally with similar biochemical functions [31]. Members of the same gene family can be closely arranged, forming a gene cluster, or can be scattered throughout the chromosome, with different patterns of expression and regulation. Gene families play an important role in the evolution and functional analysis of different species [32]. For $R$. anatipestifer, most of the high copy number gene families were annotated as hypothetical genes, with RA-CH2 having a higher copy number compared to the other strains (Figure 6A). The number of gene families in different strains reflected phenotypic differences. Copy numbers of core gene families may be related to quantitative traits, while non-core gene families may be associated with strain-specific traits. RA-CH-1 had four non-core gene families with higher copy numbers, but these were not detected using the KEGG or COG databases. Multicopy gene family analysis showed that the four strains analyzed had only six multi-copy gene families, with $\mathrm{RA}-\mathrm{CH}-2$ family members having higher copy numbers. Overall, RA-CH-1 had the most unique family members (up to 16), while the other three strains had only 1-2 unique gene families per strain (Figure 6B). RA-CH-1 had 787 unique genes, while each of the other three strains had approximately 500 unique genes each. This 


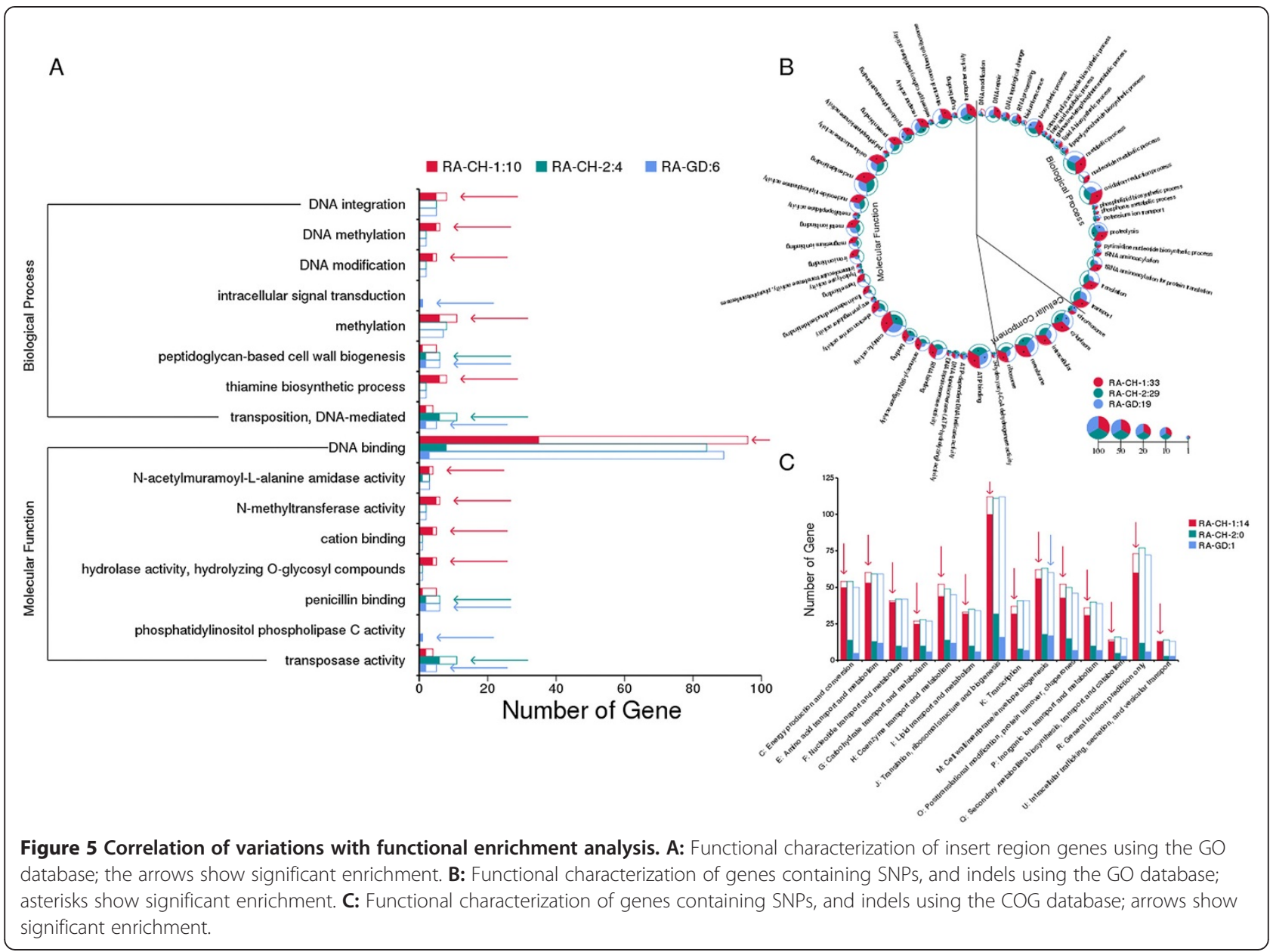

complexity could reflect the differing biological characteristics of $R$. anatipestifer strains.

\section{Phylogenetic analysis}

We constructed two phylogenetic trees of the four $R$. anatipestifer strains using conservative and nonconservative elements. The topological structures of the conservative and non-conservative trees are similar, but with different branch lengths (Figure 7). Phylogenetic analysis determined that $\mathrm{RA}-\mathrm{CH}-1$ and $\mathrm{RA}-\mathrm{CH}-2$ belong to the same branch, but the relationship of their ancestor node with the ATCC11845 and RA-GD strains was unclear (Figure 7). RA-CH-1 and RA-CH2 appear closely related to ATCC11845, but are on different branches compared to RA-GD (Figure 7). Additionally, structural analysis demonstrated that some conserved components were not in annotated CDSs. Most of the conserved components were in conserved regions and non-conserved regions had eight times the polymorphism rate of conserved regions (Figure 7). When compared to other flavobacteria, the four $R$. anatipestifer strains and R. columbina cluster in one group, while other flavobacterium belong to another group (Figure 8).

\section{Conclusions}

We successfully isolated two $R$. anatipestifer strains, RA$\mathrm{CH}-1$ and $\mathrm{RA}-\mathrm{CH}-2$, from Chengdu and Mianyang, respectively, in the SiChuan province of China, and completed their genome sequences. Using a mixture of comparative genomics strategies, we completed a comprehensive analysis of four $R$. anatipestifer strains: RA$\mathrm{CH}-1$, RA-CH-2, ATCC11845, and RA-GD, to identify factors involved in pathogenesis. Our findings will form the foundation of future investigations into the pathogenesis of $R$. anatipestifer.

\section{Methods}

\section{Genome sequencing and annotation}

Specimen collection and DNA extraction

$\mathrm{RA}-\mathrm{CH}-1$ and $\mathrm{RA}-\mathrm{CH}-2$ were isolated from the brain of sick ducks from Chengdu and Mianyang, respectively, in 

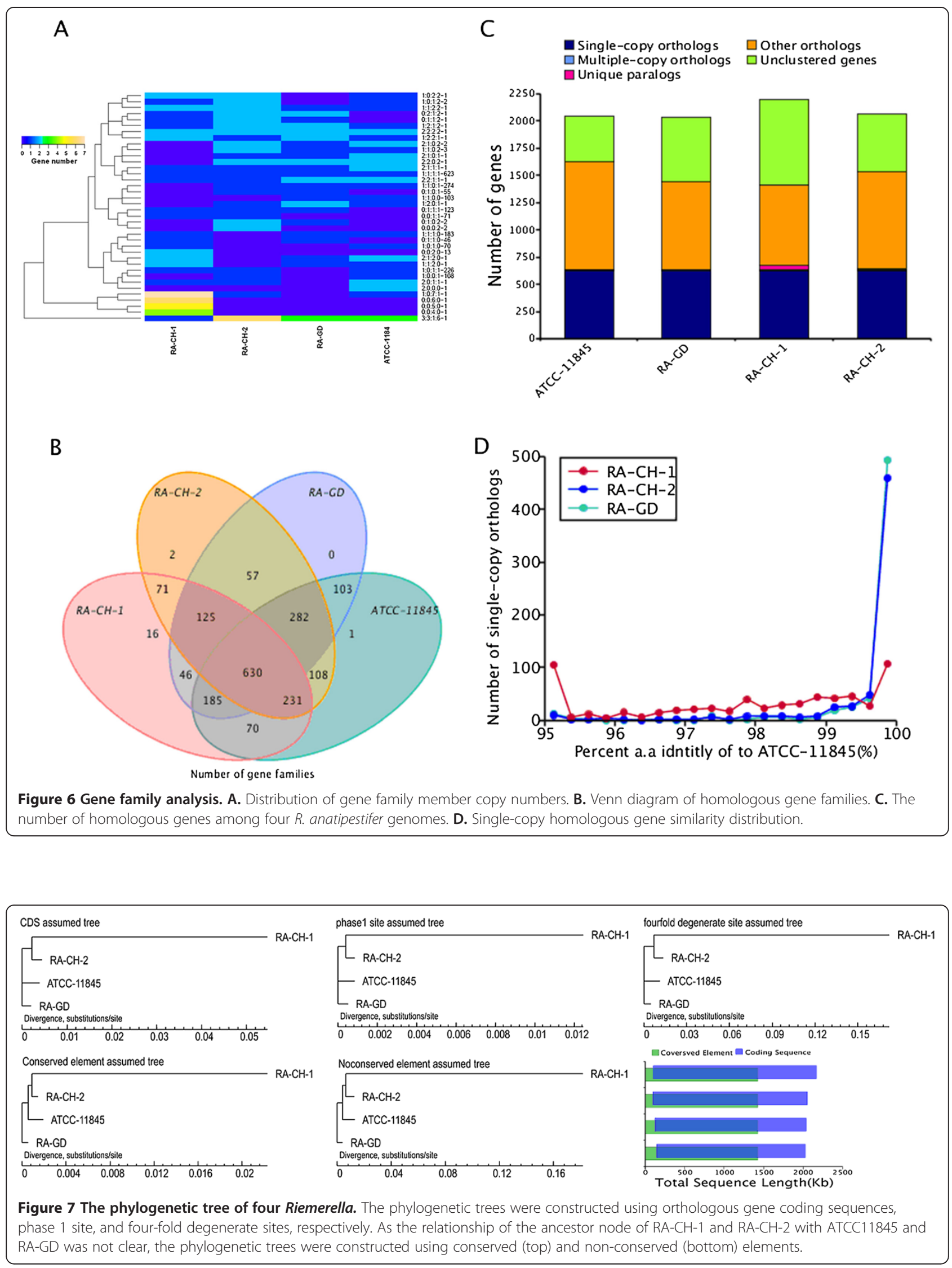


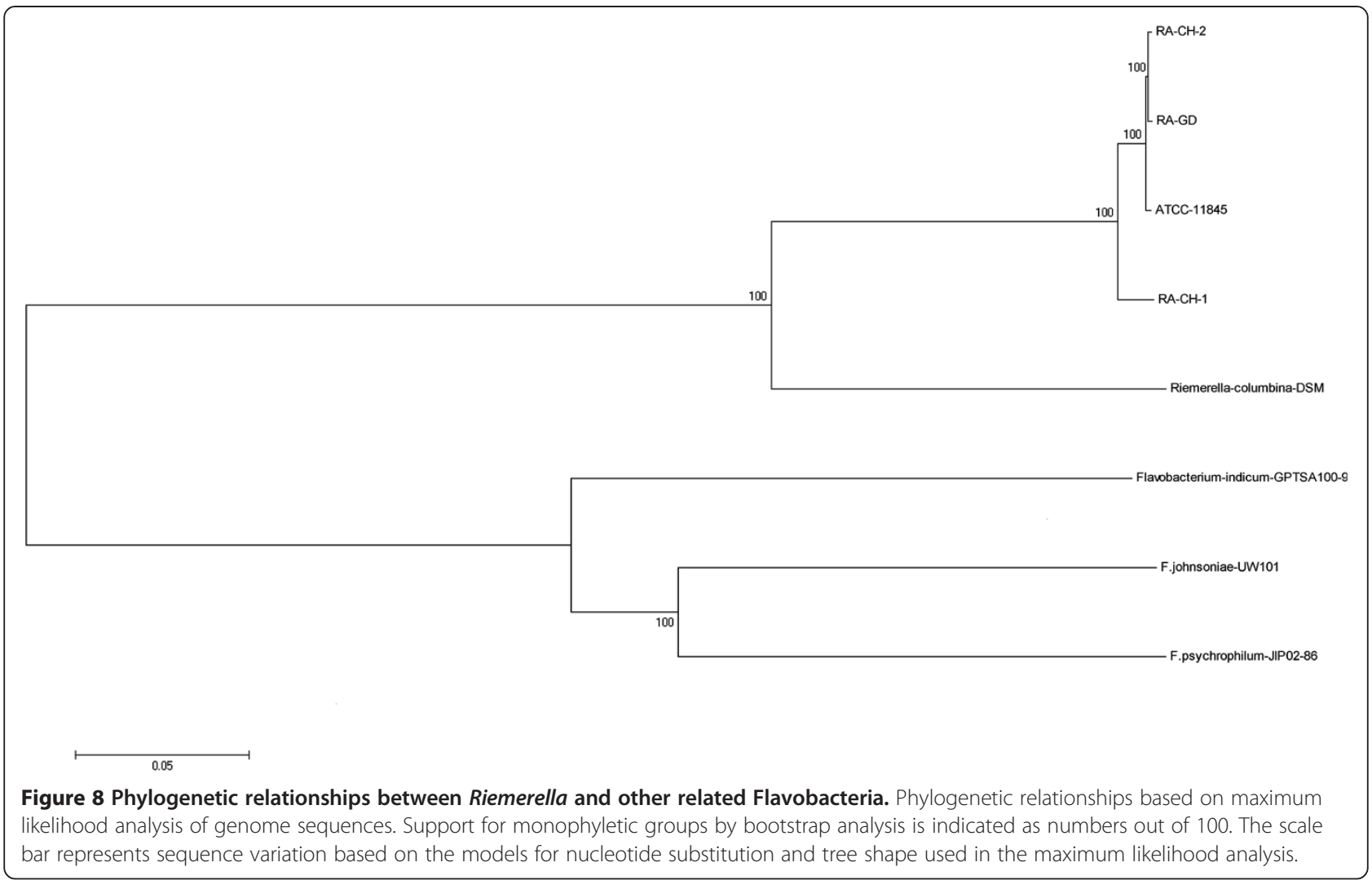

the SiChuan province of China. Serotyping were performed for RA-CH-1 and RA-CH-2 using reference serum of National Animal Disease Center. RA-CH-1 is serotype $1, \mathrm{RA}-\mathrm{CH}-2$ is serotype 2 . The samples were lyophilized after two successive transfers of stock culture on tryptic soy agar (TSA, Difco, Detroit, USA) containing $5 \%$ defibrinated sheep blood at $37^{\circ} \mathrm{C}$ for $24-48 \mathrm{~h}$. $R$. anatipestifer genomic DNA was extracted and purified via proteinase $\mathrm{K}$ treatment, multiple phenol extractions, ethanol precipitation, and spooling. Genomic DNA was checked for quality by monitoring $\mathrm{A}_{260} / \mathrm{A}_{280}$ ratios (DU800, Beckman Coulter, USA).

\section{DNA sequencing and assembly}

Bacterial strains were sequenced using an Illumina Hiseq2000 (Illumina Inc., San Diego, CA) with a multiplexed protocol. Paired-end $90 \mathrm{nt}$ long reads from $500 \mathrm{bp}$ and $6 \mathrm{~kb}$ random sequencing libraries were generated for strains $\mathrm{RA}-\mathrm{CH}-1$, and RA-CH-2. Raw data in four steps, including removing reads with $5 \mathrm{bp}$ of ambiguous bases, removing reads with 20 bp of low quality $(\leq \mathrm{Q} 20)$ bases, removing adapter contamination, and removing duplicated reads. Finally, $100 \times 500 \mathrm{bp}$ and $50 \times 6 \mathrm{~kb}$ libraries were obtained with clean paired-end read data. Assembly was performed using SOAPdenovo v1.05 [33] (http://soap.genomics.org.cn/ soapdenovo.html). The genome of the RA-GD strain was downloaded from NCBI (ftp://ftp.ncbi.nih.gov/ genbank/genomes/Bacteria/Riemerella_anatipestifer_RA_ GD_uid49039).

\section{Repetitive sequences analysis}

The genome was searched for tandem repeats using Tandem Repeats Finder [34] and Repbase [35] to identify interspersed repeats. Transposable elements in the genome assembly were identified at both the DNA and protein levels. To identify transposable elements at the DNA level, Repeat Masker was applied using a custom library based on Repbase. For protein analysis, Repeat Protein Mask from the Repeat Masker package was used to perform RMBlastX against a transposable elements protein database.

\section{Gene predict analysis}

Genes were predicted using Glimmer v3.02 [36] (http:// www.cbcb.umd.edu/software/glimmer). This software predicts start sites and coding region more effectively and has better interpolation of hidden Markov models, reducing the ratio of false positive predictions.

\section{Gene functional annotation}

Function annotation was accomplished by analysis of protein sequences. Genes were aligned with databases to 
obtain the annotation corresponding to homologs, with the highest quality alignment result chosen as the gene annotation. Function annotation was completed by comparing BLAST v2.2.23 (http://blast.ncbi.nlm.nih.gov/Blast.cgi) results in M8 format to the Kyoto Encyclopedia of Genes and Genomes (KEGG) v59 [37], Cluster of Orthologous Groups of proteins (COG) v20090331 [27,38], SwissProt v2011_10_19 [39], NR v2012-02-29, and Gene Ontology (GO) v1.419 [40] databases.

\section{Comparative genomic analyses Structural variation}

The sequences of the RA-CH-1, RA-CH-2, and RA-GD strains were compared to the reference sequence ATCC11845 using Mummer v3.22 [41] (http://mummer. sourceforge.net) for the chain stander and start side selection and LASTZ v1.01.50 [42] (http://www.bx.psu. edu/miller_lab/dist/README.lastz-1.02.00) for detailed alignment. Syntenic regions, deletions, insertions, inversions, and translocations were identified from the alignment blocks [43].

\section{SNP and small indel identification}

SNPs were identified in mismatch sites from syntenic regions. SNPs located in sequence gaps, repeat regions, or at scaffold ends were discarded. To validate the resulting non-redundant candidate SNPs, high-quality paired-end reads were mapped to the corresponding genomes with SOAPaligner v2.21 [44] (http://soap.genomics.org.cn), and the most abundant (n1) and the second most abundant (n2) nucleotides at each SNP position in each strain were examined. High quality SNPs were defined as those where the quality score of each mapped base was $>$ Q20 and that satisfied the criteria $n 1+n 2 \geq 10$ and $n 1 / n 2 \geq 5$. If more than $95 \%$ of reads had a high-quality SNP in a certain position, the SNP was included in the final set. The resulting set of unique SNPs was filtered to obtain a set of highquality SNPs present in all strains.

Raw small insertions and deletions (indels) were defined as those with a length shorter than $50 \mathrm{bp}$. These were identified as gaps from the synteny alignment. Any indels with more than one mismatch in the sequence 10 bp upstream and downstream of the indels were eliminated.

Read validation was performed on the remaining indels. Those with three or more reads which mapped to the indels-removed sequence of the subject were retained.

\section{Phylogenetic analysis}

Gene family analysis Gene families were constructed using genes from ATCC11845, RA-CH-1, RA-CH-2, and RA-GD. The current analysis is aimed at single copy gene families, which are determined by aligning protein sequences via BLAST. Gene family clustering from alignment results was performed using orthomclSoftwarev2.0.3.tar.gz [45].

Phylogenetic tree analysis Protein alignments were converted into multiple amino acids sequence alignments using Muscle v3.8.31 (http://www.drive5.com/muscle). Gene family trees were constructed from multiple sequences alignments using the ML method with Treebest v1.9.2 [46] (http://treesoft.svn.sourceforge.net/viewvc/treesoft/trunk/treebest).

\section{Functional enrichment analysis of variant gene/proteins}

Connections between all gene function variations were analyzed using the differential gene/protein function items in the COG, GO, and KEGG databases, allowing calculation of the number of corresponding COG/GO/KEGG terms. We then determined the difference between differences within each COG/GO/KEGG group and the whole genome for variant genes/proteins using the hypergeometric test [30], with a P-value $\leq 0.05$ considered significant.

\section{Competing interests}

The authors declare that they have no competing interests.

\section{Authors' contributions}

MSW and ACC conceived the study; XJW and DKZ cultured bacteria and extracted DNA; XJW, WBL, SJY, SC, XYC and LFY assembled and annotated the genomes; MFL, XJW, WBL and LFY performed bioinformatic analyses; XJW, MFL,DKZ, KFS and RYJ drafted the manuscript. All authors read and approved the final manuscript.

\section{Acknowledgments}

The research was supported by the Special Fund for Agro-scientific Research in the Public Interest (No. 201003012), the China Agricultural Research System (CARS-43-8), the National Science and Technology Support Program for Agriculture (2011BAD34B03), the Science and Technology Support Programs of Sichuan Province, China (2011ZO0034, 2011 JO0040) and the Innovative Research Team Program in Education Department of Sichuan Province (No.12TD005).

\section{Author details}

${ }^{1}$ Avian Disease Research Center, College of Veterinary Medicine of Sichuan Agricultural University, 46\# Xinkang Road, Ya'an, Sichuan 625014, P.R. China. ${ }^{2}$ BGI-Shenzhen, Shenzhen 518083, China. ${ }^{3}$ Institute of Preventive Veterinary Medicine, Sichuan Agricultural University, Chengdu, Sichuan 611130, P.R. China. ${ }^{4}$ Key Laboratory of Animal Disease and Human Health of Sichuan Province, Sichuan Agricultural University, Chengdu, Sichuan 611130, P. R. China.

Received: 31 October 2013 Accepted: 10 June 2014 Published: 17 June 2014

\section{References}

1. Segers P, Mannheim W, Vancanneyt M, De Brandt K, Hinz KH, Kersters K, Vandamme P: Riemerella anatipestifer gen. nov., comb. nov., the causative agent of septicemia anserum exsudativa, and its phylogenetic affiliation within the Flavobacterium-Cytophaga rRNA homology group. Int J Syst Bacteriol 1993, 43(4):768-776.

2. Hess C, Enichlmayr H, Jandreski-Cvetkovic D, Liebhart D, Bilic I, Hess M: Riemerella anatipestifer outbreaks in commercial goose flocks and identification of isolates by MALDI-TOF mass spectrometry. Avian Pathol 2013, 42(2):151-156.

3. Mavromatis K, Lu M, Misra M, Lapidus A, Nolan M, Lucas S, Hammon N, Deshpande S, Cheng JF, Tapia R, Han C, Goodwin L, Pitluck S, Liolios K, Pagani L, Ivanova N, Mikhailova N, Pati A, Chen A, Palaniappan K, Land M, Hauser L, 
Jefffries CD, Detter JC, Brambilla EM, Rohde M, Goker M, Gronow S, Woyke T, Bristow J, et al: Complete genome sequence of Riemerella anatipestifer type strain (ATCC 11845). Stand Genomic Sci 2011, 4(2):144-153.

4. Sarver CF, Morishita TY, Nersessian B: The effect of route of inoculation and challenge dosage on Riemerella anatipestifer infection in Pekin ducks (Anas platyrhynchos). Avian Dis 2005, 49(1):104-107.

5. Pathanasophon P, Phuektes P, Tanticharoenyos T, Narongsak W, Sawada T: A potential new serotype of Riemerella anatipestifer isolated from ducks in Thailand. Avian Pathol 2002, 31(3):267-270.

6. Christensen H, Bisgaard M: Phylogenetic relationships of Riemerella anatipestifer serovars and related taxa and an evaluation of specific PCR tests reported for R. anatipestifer. J App/ Microbiol 2010, 108(5):1612-1619.

7. Tsai HJ, Liu YT, Tseng CS, Pan MJ: Genetic variation of the ompA and $16 \mathrm{~S}$ rRNA genes of Riemerella anatipestifer. Avian Pathol 2005, 34(1):55-64

8. Huang B, Subramaniam S, Chua KL, Kwang J, Loh H, Frey J, Tan HM: Molecular fingerprinting of Riemerella anatipestifer by repetitive sequence PCR. Vet Microbiol 1999, 67(3):213-219.

9. Hu Q, Tu J, Han X, Zhu Y, Ding C, Yu S: Development of multiplex PCR assay for rapid detection of Riemerella anatipestifer, Escherichia coli, and Salmonella enterica simultaneously from ducks. J Microbiol Methods 2011, 87(1):64-69.

10. Rubbenstroth D, Ryll M, Hotzel H, Christensen H, Knobloch JK, Rautenschlein $\mathrm{S}$, Bisgaard M: Description of Riemerella columbipharyngis sp. nov., isolated from the pharynx of healthy domestic pigeons (Columba livia $f$. domestica), and emended descriptions of the genus Riemerella Riemerella anatipestifer and Riemerella columbina. Int J Syst Evol Microbiol 2013, 63(Pt 1):280-287.

11. Yu CY, Liu YW, Chou SJ, Chao MR, Weng BC, Tsay JG, Chiu CH, Ching Wu C, Long Lin T, Chang CC, Chu C: Genomic diversity and molecular differentiation of Riemerella anatipestifer associated with eight outbreaks in five farms. Avian Pathol 2008, 37(3):273-279.

12. Subramaniam S, Chua KL, Tan HM, Loh H, Kuhnert P, Frey J: Phylogenetic position of Riemerella anatipestifer based on 16S rRNA gene sequences. Int J Syst Bacteriol 1997, 47(2):562-565.

13. Zhai Z, Li X, Xiao X, Yu J, Chen M, Yu Y, Wu G, Li Y, Ye L, Yao H, Lu C, Zhang W: Immunoproteomics selection of cross-protective vaccine candidates from Riemerella anatipestifer serotypes 1 and 2. Vet Microbiol 2013, 162(2-4):850-857.

14. Subramaniam S, Huang B, Loh H, Kwang J, Tan HM, Chua KL, Frey J: Characterization of a predominant immunogenic outer membrane protein of Riemerella anatipestifer. Clin Diagn Lab Immunol 2000, 7(2):168-174.

15. Hu Q, Han X, Zhou X, Ding C, Zhu Y, Yu S: OmpA is a virulence factor of Riemerella anatipestifer. Vet Microbiol 2011, 150(3-4):278-283.

16. Weng $\mathrm{S}$, Lin W, Chang Y, Chang C: Identification of a virulence-associated protein homolog gene and ISRa1 in a plasmid of Riemerella anatipestifer. FEMS Microbiol Lett 1999, 179(1):11-19.

17. Crasta KC, Chua KL, Subramaniam S, Frey J, Loh H, Tan HM: Identification and characterization of CAMP cohemolysin as a potential virulence factor of Riemerella anatipestifer. J Bacteriol 2002, 184(7):1932-1939.

18. Wang X, Zhu D, Wang M, Cheng A, Jia R, Zhou Y, Chen Z, Luo Q, Liu F, Wang $Y$, Chen $X$ : Complete genome sequence of Riemerella anatipestifer reference strain. J Bacteriol 2012, 194(12):3270-3271.

19. Tu J, Lu F, Miao S, Ni X, Jiang P, Yu H, Xing L, Yu S, Ding C, Hu Q: The siderophore-interacting protein is involved in iron acquisition and virulence of Riemerella anatipestifer strain CH3. Vet Microbiol 2014, 168(2-4):395-402.

20. Lu F, Miao S, Tu J, Ni X, Xing L, Yu H, Pan L, Hu Q: The role of TonBdependent receptor TbdR1 in Riemerella anatipestifer in iron acquisition and virulence. Vet Microbiol 2013, 167(3-4):713-718.

21. McBride MJ, Xie G, Martens EC, Lapidus A, Henrissat B, Rhodes RG, Goltsman E, Wang W, Xu J, Hunnicutt DW, Staroscik AM, Hoover TR, Cheng YQ, Stein $\mathrm{L} L$ : Novel features of the polysaccharide-digesting gliding bacterium Flavobacterium johnsoniae as revealed by genome sequence analysis. Appl Environ Microbiol 2009, 75(21):6864-6875.

22. Xie G, Bruce DC, Challacombe JF, Chertkov O, Detter JC, Gilna P, Han CS, Lucas S, Misra M, Myers GL, Richardson P, Tapia R, Thayer N, Thompson LS, Brettin TS, Henrissat B, Wilson DB, McBride MJ: Genome sequence of the cellulolytic gliding bacterium Cytophaga hutchinsonii. Appl Environ Microbiol 2007, 73(11):3536-3546.

23. McBride MJ, Zhu Y: Gliding motility and Por secretion system genes are widespread among members of the phylum bacteroidetes. $J$ Bacteriol 2013, 195(2):270-278.
24. Sato K, Naito M, Yukitake H, Hirakawa H, Shoji M, McBride MJ, Rhodes RG, Nakayama K: A protein secretion system linked to bacteroidete gliding motility and pathogenesis. Proc Natl Acad Sci U S A 2010, 107(1):276-281.

25. Saiki K, Konishi K: Identification of a Porphyromonas gingivalis novel protein sov required for the secretion of gingipains. Microbiol Immunol 2007, 51(5):483-491.

26. Braun TF, Khubbar MK, Saffarini DA, McBride MJ: Flavobacterium johnsoniae gliding motility genes identified by mariner mutagenesis. J Bacteriol 2005, 187(20):6943-6952.

27. Tatusov RL, Fedorova ND, Jackson JD, Jacobs AR, Kiryutin B, Koonin EV, Krylov DM, Mazumder R, Mekhedov SL, Nikolskaya AN, Rao BS, Smirnov S, Sverdlov AV, Vasudevan S, Wolf YI, Yin JJ, Natale DA: The COG database: an updated version includes eukaryotes. BMC Bioinformatics 2003, 4:41.

28. Kanehisa M, Goto S, Kawashima S, Okuno Y, Hattori M: The KEGG resource for deciphering the genome. Nucleic Acids Res 2004, 32(Database issue):D277-D280.

29. Karp PD, Paley S, Romero P: The pathway tools software. Bioinformatics 2002, 18(Suppl 1):S225-S232

30. Zhou X, Su Z: EasyGO: gene ontology-based annotation and functional enrichment analysis tool for agronomical species. BMC Genomics 2007, 8:246.

31. Demuth JP, De Bie T, Stajich JE, Cristianini N, Hahn MW: The evolution of mammalian gene families. PloS One 2006, 1:e85.

32. Skinner MK, Rawls A, Wilson-Rawls J, Roalson EH: Basic helix-loop-helix transcription factor gene family phylogenetics and nomenclature. Differentiation 2010, 80(1):1-8.

33. Li R, Li Y, Kristiansen K, Wang J: SOAP: short oligonucleotide alignment program. Bioinformatics 2008, 24(5):713-714.

34. Benson G: Tandem repeats finder: a program to analyze DNA sequences. Nucleic Acids Res 1999, 27(2):573-580.

35. Jurka J, Kapitonov W, Pavlicek A, Klonowski P, Kohany O, Walichiewicz J: Repbase update, a database of eukaryotic repetitive elements. Cytogenet Genome Res 2005, 110(1-4):462-467.

36. Delcher AL, Harmon D, Kasif S, White O, Salzberg SL: Improved microbial gene identification with GLIMMER. Nucleic Acids Res 1999, 27(23):4636-4641.

37. Kanehisa M, Goto S, Hattori M, Aoki-Kinoshita KF, Itoh M, Kawashima S, Katayama T, Araki M, Hirakawa M: From genomics to chemical genomics: new developments in KEGG. Nucleic Acids Res 2006, 34(Database issue):D354-D357.

38. Tatusov RL, Koonin EV, Lipman DJ: A genomic perspective on protein families. Science 1997, 278(5338):631-637.

39. Magrane M, Consortium U: UniProt knowledgebase: a hub of integrated protein data. Database (Oxford) 2011, 2011:bar009.

40. Ashburner M, Ball CA, Blake JA, Botstein D, Butler H, Cherry JM, Davis AP, Dolinski K, Dwight SS, Eppig JT, Harris MA, Hill DP, Issel-Tarver L, Kasarskis A, Lewis S, Matese JC, Richardson JE, Ringwald M, Rubin GM, Sherlock G: Gene ontology: tool for the unification of biology: the gene ontology consortium. Nat Genet 2000, 25(1):25-29.

41. Kurtz S, Phillippy A, Delcher AL, Smoot M, Shumway M, Antonescu C, Salzberg SL: Versatile and open software for comparing large genomes. Genome Biol 2004, 5(2):R12.

42. Dewey CNPL: Evolution at the nucleotide level: the problem of multiple whole-genome alignment. Hum Mol Genet 2006, 15(1):R51-R56.

43. Li Y, Zheng H, Luo R, Wu H, Zhu H, Li R, Cao H, Wu B, Huang S, Shao H, Ma H, Zhang F, Feng S, Zhang W, Du H, Tian G, Li J, Zhang X, Li S, Bolund L, Kristiansen K, de Smith AJ, Blakemore Al, Coin LJ, Yang H, Wang J, Wang J: Structural variation in two human genomes mapped at singlenucleotide resolution by whole genome de novo assembly. Nat Biotechnol 2011, 29(8):723-730.

44. Li R, Li Y, Fang $X$, Yang H, Wang J, Kristiansen $K$, Wang J: SNP detection for massively parallel whole-genome resequencing. Genome Res 2009, 19(6):1124-1132

45. Li L, Stoeckert CJ Jr, Roos DS: OrthoMCL: identification of ortholog groups for eukaryotic genomes. Genome Res 2003, 13(9):2178-2189.

46. Nandi T, Ong C, Singh AP, Boddey J, Atkins T, Sarkar-Tyson M, Essex-Lopresti $\mathrm{AE}$, Chua HH, Pearson T, Kreisberg JF, Nilsson C, Ariyaratne P, Ronning C, Losada L, Ruan Y, Sung WK, Woods D, Titball RW, Beacham I, Peak I, Keim P, Nierman WC, Tan P: A genomic survey of positive selection in Burkholderia pseudomallei provides insights into the evolution of accidental virulence. PLoS Pathogens 2010, 6(4):e1000845.

doi:10.1186/1471-2164-15-479

Cite this article as: Wang et al: Comparative genomics of Riemerella anatipestifer reveals genetic diversity. BMC Genomics 2014 15:479. 